\title{
Pericarditis purulenta por Staphylococcus aureus sin foco en paciente con neoplasia pancreática
}

Pablo López-Quirantes, Jaime Sanz-Cánovas, María López-Sánchez, Ricardo Gómez-Huelgas

Servicio de Medicina Interna. Hospital Regional Universitario de Málaga. Málaga. España

Citar como: López-Quirantes P, Sanz-Cánovas J, López-Sánchez M, Gómez-Huelgas R. Pericarditis purulenta por Staphylococcus aureus sin foco en paciente con neoplasia pancreática. Rev Esp Casos Clin Med Intern (RECCMI). 2020 (Ago); 5(2): 84-86. doi: 10.32818/reccmi.a5n2a10.

Cite this as: López-Quirantes P, Sanz-Cánovas J, López-Sánchez M, Gómez-Huelgas R. Purulent Staphylococcus aureus pericarditis without focus in a patient with pancreatic neoplasia. Rev Esp Casos Clin Med Intern (RECCMI). 2020 (Ago); 5(2): 84-86. doi: 10.32818/reccmi.a5n2a10.

Autor para correspondencia: Jaime Sanz-Cánovas. jaimesc25@hotmail.com

\section{Palabras clave \\ $\triangleright$ Pericarditis purulenta \\ $\triangleright$ Staphylococcus aureus \\ $\triangleright$ Neoplasia pancreática}

$\triangleright$ Pericardiectomía

\section{Keywords}

$\triangleright$ Purulent pericarditis

$\triangleright$ Staphylococcus aureus

$\triangleright$ Pancreatic neoplasm

$\triangleright$ Pericardiectomy

\section{Resumen}

Presentamos el caso de un varón con cuadro febril y disnea de corta duración, diagnosticado de pericarditis purulenta con bacteriemia asociada por Staphylococcus aureus, en el que no se encontró foco primario infeccioso. Como hallazgo casual, se le diagnosticó de neoplasia pancreática. Tras someterse a intervención quirúrgica, comenzó con inestabilidad hemodinámica y mala evolución a pesar de las medidas intensivas, y falleció durante el posoperatorio.

Abstract
We present the case of a patient with a short-term fever and dyspnea, diagnosed with purulent pericarditis
with bacteremia associated with Staphylococcus aureus, and no primary infectious focus found. As a casual
finding, a pancreatic neoplasm was detected. After undergoing surgery, he developed hemodynamic instability
and poor evolution despite intensive measures and died during the postoperative period.

\section{Puntos destacados}

$\triangleright$ Exponemos un caso de pericarditis purulenta, entidad poco frecuente actualmente, en la que no se detectó un antecedente infeccioso primario ni intervención quirúrgica previa, como suele ser habitual en este cuadro.

\section{Introducción}

Hoy en día, la pericarditis purulenta es una entidad muy poco frecuente gracias a la amplia disponibilidad de antibióticos existente. A pesar de ello, existen casos en los que la alta sospecha clínica y el tratamiento precoz son clave para evitar complicaciones graves, e incluso la muerte del paciente.

\section{Caso clínico}

\section{Antecedentes}

Varón de 57 años que vivía en medio urbano con su pareja. No presentaba alergias medicamentosas conocidas. Fumador activo de 1 paquete/día y bebedor de 2 cervezas/día, con datos de hepatopatía crónica con hipertensión portal sin descompensaciones previas. Diabetes mellitus tipo 2 en tratamiento con sitagliptina y buen control metabólico (HbA1c 6,6\%).

\section{Enfermedad actual y pruebas complementarias}

Acudía a Urgencias por fiebre de hasta 39,7 C, dolor torácico de características atípicas y disnea de mínimos esfuerzos de 72 horas de evolución. La semana previa presentó cuadro catarral ya resuelto. Negaba otras manifestaciones clínicas a nivel neurológico, cutáneo, digestivo o urinario. Se extrajeron hemocultivos.

A su ingreso en planta, presentaba una temperatura de $37,6^{\circ} \mathrm{C}$, presión arterial $110 / 70 \mathrm{mmHg}$ y frecuencia cardíaca $70 \mathrm{lpm}$, manteniendo saturaciones basales de $\mathrm{O}_{2}$ del $96 \%$. No se apreciaron signos de interés y en la auscultación cardíaca presentaba tonos apagados y rítmicos, sin apreciarse roce pericárdico.

En la analítica de Urgencias destacaban leucocitosis con desviación izquierda (leucocitos $21.620 / \mathrm{mm}^{3}$, neutrófilos $19.030 / \mathrm{mm}^{3}$ ) y elevación de la PCR $(171 \mathrm{mg} / \mathrm{l})$, troponina I (6.728 ng/l) y del dímero-D $(4.187 \mathrm{ng} / \mathrm{ml})$.

En la radiografía de tórax, se apreciaba cardiomegalia global no presente en estudios previos (Figura 1). La PCR SARS-CoV-2 fue negativa. El electrocardio- 
López-Quirantes P, Sanz-Cánovas J, López-Sánchez M, Gómez-Huelgas R. Pericarditis purulenta por Staphylococcus aureus $\sin$ foco en paciente con neoplasia pancreática

grama mostró buenos voltajes y un ritmo sinusal a unos 75 lpm, con ascenso del segmento ST en II, III, aVF y V3-V6, sin observarse alternancia eléctrica. Se realizó una ecocardioscopia urgente, visualizándose un derrame pericárdico severo (sin signos indirectos de compromiso hemodinámico). Una angio-TC torácica descartó tromboembolismo pulmonar.

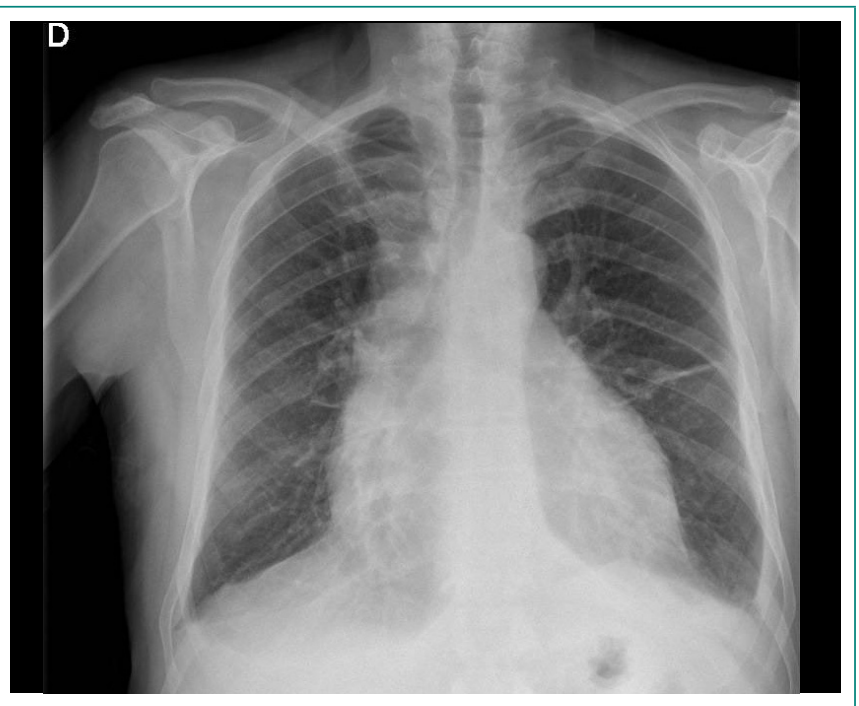

Figura 1. Radiografía de tórax

\section{Evolución}

El paciente fue ingresado en el Servicio de Medicina Interna con sospecha de pericarditis aguda con derrame pericárdico, iniciándose antibioterapia empírica con ceftriaxona y linezolid.

Se solicitó analítica de sangre con serología, autoinmunidad y marcadores tumorales, obteniéndose todos los parámetros en rango normal, a excepción de una discreta elevación del Ca 19.9. Al mismo tiempo, se realizó PET-TC para descartar un origen paraneoplásico, observándose un foco hipermetabólico de moderada intensidad (SUVmáx 4,37) en cuerpo/cola pancreática. Una TC de abdomen con contraste confirmó una posible neoplasia pancreática de unos $14 \mathrm{~mm}$, sin otros hallazgos.

Se realizó pericardiocentesis evacuadora, en la que se extrajeron $800 \mathrm{ml}$ de líquido purulento. El análisis del líquido pericárdico fue compatible con exudado (leucocitos 17.150/ $\mathrm{mm}^{3}$, neutrófilos $90 \%$, glucosa < $1 \mathrm{mg} / \mathrm{dl}$, proteínas totales $6 \mathrm{~g} / \mathrm{dl}$ ). Se enviaron muestras para cultivo, creciendo a las 48 horas colonias de Staphylococcus aureus meticilin-sensible que se aislaron también en los hemocultivos.

Se instauró antibioterapia dirigida con cloxacilina y levofloxacino. A las 72 horas, se realizó un nuevo ecocardiograma transtorácico de control, objetivándose tabicaciones y persistencia del derrame pericárdico purulento sin repercusión cardíaca.

Tras presentar el caso en sesión médico-quirúrgica, el paciente se sometió a una pericardiectomía anterior amplia. Durante la intervención se apreció un pericardio engrosado, un epicardio con intensos signos inflamatorios y persistencia del derrame pericárdico seropurulento. El estudio anatomopatológico mostró una inflamación aguda severa abscesificada, compatible con pericarditis aguda fibrinoide.

Tras la cirugía, el paciente pasó a la Unidad de Cuidados Intensivos, donde desarrolló un shock hemodinámico refractario, presentando altos débitos pericárdicos (hasta 2,5 litros de drenaje hemático). A pesar de las medidas intensivas de soporte hemodinámico y ventilatorio, el paciente falleció a las 8 horas de la intervención.

\section{Diagnóstico}

Pericarditis purulenta por Staphylococcus aureus.

\section{Discusión y conclusión}

La pericarditis purulenta, o infección del espacio pericárdico con producción purulenta, es una entidad muy poco frecuente en la era moderna desde que disponemos de antibióticos, y tiene actualmente una incidencia muy baja'. Las formas primarias son infrecuentes, siendo más comunes las pericarditis purulentas secundarias por distintos mecanismos: extensión por continuidad de un foco intratorácico o miocárdico, diseminación hematógena o debida a lesiones perforantes o cirugía².

Los factores predisponentes para la pericarditis purulenta han cambiado con el tiempo. En la era previa a los antibióticos, solían asociarse a una enfermedad infecciosa subyacente, la mayor parte de origen respiratorio, y menos habitualmente a endocarditis, meningitis o foco infeccioso cutáneo u ótico². Actualmente, los pacientes con pericarditis purulenta suelen presentar otros factores predisponentes, como una cirugía torácica reciente, la realización de técnicas percutáneas invasivas (inyección con aguja endobronquial, escleroterapia endoscópica de varices esofágicas, fundoplicatura laparoscópica de Nissen, fibrobroncoscopia o angioplastia coronaria) o la presencia de comorbilidades (enfermedad renal crónica, inmunosupresión, alcoholismo, artritis reumatoide o malignidad) ${ }^{3-5}$.

Los microorganismos más prevalentes son los estreptococos (principalmente en casos por contigüidad intratorácica) y los estafilococos (mayormente por vía hematógena). En menor medida, se han descrito casos por gramnegativos y anaerobios, así como por hongos como Candida spp en presencia de inmunosupresión ${ }^{5}$. En nuestro medio, una revisión retrospectiva de un hospital español desde 1971 hasta 1991 registró 33 casos de pericarditis purulenta confirmada, de los que 14 se diagnosticaron en la autopsia. La mayoría de los casos presentó un foco respiratorio previo, en gran parte con empiema asociado, con predominio de estreptococos como principal microorganismo'.

La clínica de la pericarditis purulenta es aguda, caracterizada por fiebre elevada con escalofríos (96\%), mientras que el dolor torácico y el roce pericárdico sólo están presentes en menos del 30\% y del 50\% de los casos, respectivamente 2 . Son comunes la anemia y la leucocitosis con desviación izquierda y, con frecuencia, una radiografía de tórax revela derrame pleural y ensanchamiento mediastínico. El electrocardiograma puede ser normal hasta en el 35\% de los casos.

Aunque el ecocardiograma transtorácico facilita la detección y cuantificación del derrame pericárdico, el diagnóstico definitivo depende del análisis del líquido pericárdico. El aspecto macroscópico purulento junto con el cultivo positivo son definitorios. El análisis del líquido muestra leucocitosis polimorfonuclear, niveles bajos de glucosa y proteínas con niveles elevados de lactato deshidrogenasa ${ }^{2,5}$. Suele ser frecuente un diagnóstico tardío por la ausencia de características típicas de la pericarditis y la tendencia a atribuir los síntomas, inicialmente, a otros procesos ${ }^{1}$.

El tratamiento se basa en el uso de antibioterapia con cobertura para microorganismos grampositivos y gramnegativos durante 4 semanas hasta obtener el resultado de los cultivos junto con el drenaje del espacio pericárdico. En ocasiones, la tabicación del espacio pericárdico y el acúmulo de fibrina conllevan un escaso débito tras la pericardiocentesis y predispone a pericarditis constrictiva. Por ello, en estos casos es necesaria la realización de una ventana 
pericárdica o una pericardiectomía. Aunque esta última facilita mejor evacuación del pus y menor probabilidad de evolucionar a pericarditis constrictiva, la complejidad y el riesgo de la intervención son mayores ${ }^{6}$. Está indicada cuando encontramos adherencias densas, tabiques, mala evolución infecciosa o compromiso cardíaco?.

Sin tratamiento, la mortalidad de esta patología ronda el 100\%4. Aun con el manejo adecuado, la mortalidad se acerca al 40\%. Las principales complicaciones incluyen el taponamiento cardíaco, la evolución a pericarditis constrictiva, la formación de aneurismas micóticos (sobre todo por estafilococos y salmonela) o pseudoaneurismas en el ventrículo izquierdo'.

Como conclusión, la pericarditis purulenta es una entidad poco frecuente en la actualidad. Precisa un alto índice de sospecha clínica para poder llevar a cabo un manejo adecuado, ya que presenta una mortalidad elevada.

\section{Bibliografía}

1. Sagristà-Sauleda J, Barrabés JA, Permanyer-Miralda G, Soler-Soler J. Purulent pericarditis: review of a 20-year experience in a general hospital.
J Am Coll Cardiol. 1993; 22(6): 1661-1665. doi: 10.1016/0735-1097(93)90592-o

2. Rubin RH, Moellering RC Jr. Clinical, microbiologic and therapeutic aspects of purulent pericarditis. Am J Med. 1975; 59(1): 68-78. doi: 10.1016/00029343(75)90323-x

3. Permanyer-Miralda G, Sagristá-Sauleda J, Soler-Soler J. Primary acute pericardial disease: a prospective series of 231 consecutive patients. Am J Cardiol. 1985; 56(10): 623-630. doi: 10.1016/0002-9149(85)91023-9.

4. Klacsmann PG, Bulkley BH, Hutchins GM. The changed spectrum of purulent pericarditis: an 86 year autopsy experience in 200 patients. Am J Med. 1977; 63(5): 666-673. doi:10.1016/0002-9343(77)90150-4.

5. Parikh SV, Memon N, Echols M, Shah J, McGuire DK, Keeley EC. Purulent pericarditis: report of 2 cases and review of the literature. Medicine (Baltimore). 2009; 88(1): 52-65. doi: 10.1097/MD.0b013e318194432b.

6. Cui HB, Chen XY, Cui CC, Shou XL, Liu XH, Yao XW, Wang JK, Guan GC. Prevention of pericardial constriction by transcatheter intrapericardial fibrinolysis with urokinase. Chin Med Sci J. 2005; 20(1): 5-10.

7. Adler Y, Charron P, Imazio M, Badano L, Barón-Esquivias G, Boaert J, et al. Guía ESC 2015 sobre el diagnóstico y tratamiento de las enfermedades del pericardio. Rev Esp Cardiol. 2015; 68(12): 1126.e-24. doi: 10.1016/j.recesp.2015.10.011 